\begin{tabular}{l}
\hline Alimentary Pharmacology \\
\hline \& Therapeutics \\
\hline
\end{tabular}

\title{
Depression and treatment with antidepressants are associated with the development of gastroesophageal reflux disease
}

\begin{tabular}{|r|l|}
\hline Journal: & Alimentary Pharmacology \& Therapeutics \\
\hline Manuscript ID: & APT-0052-2010.R1 \\
\hline Manuscript Type: & Original Scientific Paper \\
\hline Date Submitted by the & 23-Feb-2010 \\
\hline Complete List of Authors: & $\begin{array}{l}\text { Martín-Merino, Elisa; Centro Español de Investigación } \\
\text { Farmacoepidemiológica (CEIFE) } \\
\text { Ruigomez, Ana; CEIFE, none } \\
\text { Garcia Rodriguez, Luis Alberto; Centro Español de Investigacion } \\
\text { Farmacoepidemiologica (CEIFE) } \\
\text { Wallander, Mari-Ann; Uppsala University, Department of Public } \\
\text { Health and Caring Sciences } \\
\text { Johansson, Saga; AstraZeneca R\&D; Gothenburg University, } \\
\text { Institute of Medicine, Sahlgrenska Academy }\end{array}$ \\
\hline Keywords: & $\begin{array}{l}\text { GERD or GORD < Disease-based, Epidemiology < Topics, General } \\
\text { practice < Topics, Psychiatric disorders < Topics }\end{array}$ \\
\hline
\end{tabular}

\section{s ScholaroNE"


Spanish Centre for Pharmacoepidemiologic Research (CEIFE)

Almirante $28\left(2^{\circ}\right)$

Madrid 28004

Spain

Tel: 0034915311485

Fax: 0034915312871

Email: aruigomez@ceife.es

23 February 2010

Professor Roy E Pounder

Editor

c/o Alimentary Pharmacology \& Therapeutics Editorial Office

1 Chestnut Walk

Oaklands

Welwyn

Herts

AL6 0SB

Dear Professor Pounder,

Thank you very much for giving us the opportunity to revise our manuscript 'Depression and treatment with antidepressants are associated with the development of gastroesophageal reflux disease' (Manuscript ID APT-0052-2010) for consideration for publication in Alimentary Pharmacology \& Therapeutics.

We thank the reviewers for their helpful comments and have revised the manuscript accordingly. Please find a point-by-point response to the reviewers' comments below. 
We hope you find that the revised manuscript is now acceptable for publication in your journal. Please do not hesitate to contact me if there is anything else you require. Thank you for considering our manuscript, and I look forward to hearing from you.

Yours sincerely,

Elisa Martín-Merino

Response to reviewers - Manuscript APT-0052-2010

\section{Editor's comments to author:}

Comment: We are not very keen on Titles which pose a question. You do have a conclusion to your study, so why not, "Depression and treatment with antidepressants are associated with the development of gastroesophageal reflux disease".

Response: We have now changed the title.

Comment: In the Summary, your work is correctly in the past tense, but your Conclusion should be in the present tense.

Response: We have changed this to present tense.

\section{Reviewers' comments to author:}

Reviewer: 1

Comment: The modelling was a little hard to follow. I wasn't sure the association of depression and GERD held up if medications including antidepressants, smoking, BMI etc. were all adjusted for in the final model. Or is the association of depression with GERD ALL largely explained by antidepressant medications?

Response: The final model to estimate the risk of GERD associated with a diagnosis of depression was adjusted by sex, age, BMI, calendar year, smoking, alcohol use and PCP visits. As shown in Table 1, the risk of GERD associated with depression in this model 
was 1.31. This model did not include antidepressant drugs because we considered this to be an indirect measure of depression status, which would dilute the risk associated with depression. If antidepressant treatment status was included in the model, the risk of GERD associated with depression decreased to 1.15 (95\% CI: 1.01-1.31). As reported in the manuscript, when all medical treatments were taken as a whole we found no difference in the incidence of GERD between treated and non-treated patients with depression. To more directly examine the interplay between depression and antidepressant treatment, we performed the following analyses (see table below). This suggests that the risk of GERD associated with a diagnosis of depression and the risk associated with antidepressant treatment are independent of each other.

\begin{tabular}{|l|c|c|c|c|c|c|}
\hline \multirow{2}{*}{ Interaction } & \multicolumn{2}{|c|}{ Controls } & \multicolumn{2}{c|}{$\begin{array}{c}\text { GERD } \\
\text { Cases }\end{array}$} & OR & 95\% CI \\
\cline { 2 - 7 } & $\mathbf{n}$ & $\%$ & No. & \% & & \\
\hline $\begin{array}{l}\text { No depression diagnosis \& not } \\
\text { exposed to antidepressants }\end{array}$ & 4644 & 46.4 & 997 & 32.5 & 1 & - \\
\hline $\begin{array}{l}\text { Depression diagnosis but no } \\
\text { antidepressant treatment }\end{array}$ & 590 & 5.9 & 223 & 7.3 & 1.50 & $1.26-1.78$ \\
\hline $\begin{array}{l}\text { Current or recent } \\
\text { antidepressant use but no } \\
\text { depression diagnosis recorded }\end{array}$ & 158 & 1.6 & 64 & 2.1 & 1.52 & $1.12-2.06$ \\
\hline $\begin{array}{l}\text { Depression diagnosis and } \\
\text { current or recent } \\
\text { antidepressant treatment }\end{array}$ & 2539 & 25.4 & 1022 & 33.4 & 1.45 & $1.30-1.61$ \\
\hline Remaining & 2069 & 20.7 & 758 & 24.8 & 1.34 & $1.19-1.50$ \\
\hline
\end{tabular}

OR adjusted by sex, smoking, PCP visits, BMI, alcohol use, age at index, calendar year and depression and antidepressant drugs.

Current use: during the 30 days prior to index date.

Recent use: use from 31 days to 365 days before index date

We have now included these results in the manuscript and added a comment in discussion (please see second paragraph of discussion).

\section{Reviewer: 2}

General comments:

This is a very interesting and well-designed study of depression and treatment with antidepressants in relation to GERD. 
Major and minor comments for each section:

Title \& Abstract:

Comment: The terminology used is somewhat confusing as there are two different study designs and statistical analyses included. To clarify, please revise the last sentence of the Methods section in the Abstract as "Furthermore, we performed a nested case-control study where odds ratios with $95 \%$ confidence intervals were estimated by unconditional logistic regression in multivariable models...".

Response: We have changed the sentence accordingly.

\section{Introduction:}

Comment: Paragraph 3, page 3 and Methods section: As reference \#5 is "in press", where detailed descriptions of THIN and the cohorts are found I cannot read this reference, but Figure 1 is helpful (but please revise "case-control analysis" into "nested case-control studies"). Was there a reason for not using one cohort and assign exposure (i.e. diagnosis of depression) for each cohort member instead of two cohorts?

Response: We have revised Figure 1 in line with this comment.

In our previous manuscript (which is now published; Prim Care Companion J Clin Psychiatry 2010; 12: e1-e8.) we described the methods used to ascertain the two study cohorts in detail. These are shown below for the reviewer's information:

"We identified all individuals who were aged 10-79 years and registered with THIN between 1 January 2002 and 31 December 2004 (the study period). These individuals were only included in the source population for follow-up if they had been registered for at least two years with their PCP prior to the start of the study period. Individuals aged 70 years or older who had fewer than two PCP visits during the follow-up period were excluded (proxy for incomplete data recorded). The date at which all the above eligibility criteria were met was defined as the patient's start date. Finally, all individuals with a recorded diagnosis of depression before the start date were excluded. After these exclusions, the source population numbered 1287829 individuals.

Identification of the depression cohort

All members of the source population were followed from the start date until the earliest of the following end points: diagnosis of depression (identified from Read codes), 
death, reaching the age of 80 years, or end of the study period (31 December 2004). We initially identified 53483 patients who received their first diagnosis of depression during the study period. Of these, we excluded patients who had five or more prescriptions for antidepressants before the recorded diagnosis, because such treatment patterns were highly unlikely to represent incident cases (6313 individuals). The final depression cohort therefore comprised 47170 individuals. The index date in this population was the date of depression diagnosis.

\section{Identification of a comparison cohort}

All members of the source population who did not have a recorded diagnosis of depression were assigned a random date within the study period as an index date. If this index date fell within the individual's period of contribution to the study, they were eligible for inclusion in the comparison cohort. The same eligibility criteria as for the depression cohort were applied, with the added condition of having no diagnosis of depression before 31 December 2004. From the pool of eligible individuals a sample was selected and frequency matched to the depression cohort by age ( \pm 1 year), sex and calendar year. The size of this comparison cohort was 50000 individuals. Each individual in the comparison cohort was assigned a random index date."

Because we had already identified these two study cohorts for our previous study we decided to use both to better estimate the association between depression and GERD. We started following up these two cohorts (one of them being a cohort of incident GERD) to ascertain the hazard rate of incident GERD as well as the hazard ratio associated with depression. Finally, we performed a nested case-control study to adjust by other potential time-dependent risk factors using all GERD cases identified in both cohorts together with a series of controls sampled among both cohorts.

\section{Comment: Not clear why other risk factors, other than depression and treatment with antidepressants, were evaluated as this is described in less detail or not at all in the Introduction and Material and Methods section and mentioned briefly in the Discussion, although the findings are very interesting.}

Response: We thank the reviewer for their encouraging comments about the results. We have now amended the last paragraph of the Introduction to clarify that the study of other risk factors was one of the key aims of the study. We have also added more detail to the 
Materials and Methods and Results sections to explain how the information on other risk factors was collected.

\section{Material and Methods:}

Comment: Analysis, page 5: Please add "Statistical" to the heading "Analysis".

However, it is somewhat confusing that additional nested case-control study design are described in this paragraph and the terminology used is also somewhat confusing. The sentence starting "A nested case-control analysis..." should be omitted and instead it should say "Furthermore, we performed a nested case-control study to be able to adjust for potential confounders such as medication use etc...". Response: We have now revised the Materials and Methods section of the manuscript including the explanation of the nested case-control study. We have also changed the terminology as suggested.

Comment: Rewrite the sentence starting "A second nested case-control analysis..." into "A second nested case-control study was performed using only the depression cohort to evaluate...". How many cases and controls were included in the second nested case-control study?

Response: We have revised the last paragraph of the Materials and Methods section in line with this comment.

Comment: Revise the last sentence into "In both nested case-control studies we used unconditional logistic regression to estimate odds ratios...AND 95\% confidence intervals??...". Omit "of a diagnosis of GERD".

Response: We have now edited the sentence as suggested by the reviewer.

Comment: Please add what statistical software(s) was/were used (SAS, Stata, etc?). Response: STATA-10.1 was used for all the statistical analysis. We have now added this information to the manuscript.

Comment: Page 6: Please use "sex" instead of "gender" (as in the Results section). 
Response: We have made this change.

Results and Tables:

Comment: Paragraph 3, page 7: Why use "hazard ratio" instead of "risk estimate" or "point estimate". Hazard ratio has not been mentioned previously.

Response: We have now added the following sentence to the analysis section to clarify this issue: "Cox regression analysis was used to estimate the hazard ratio (HR) of GERD in patients with a diagnosis of depression compared with individuals with no depression diagnosis adjusted by age and sex."

\section{Discussion:}

Comment: Well-written and interesting discussion. A strength that those with prior GERD could be excluded, but how far back in time has the THIN database recorded GERD (and other) diagnoses?

Response: Information has been entered prospectively since the late 1980s in a few practices and since the 1990s in the majority of other practices. Primary care practitioners have the option to enter as much information retrospectively as they consider fit when they start entering information prospectively.

Comment: Paragraph 2, page 10: Use "multivariable" instead of "multivariate" (not a multilevel analysis).

Response: We have now made this change.

Comment: Paragraph 2, page 11: It is very interesting that this study reveals that the link between sleep disorders and depression and GERD is bidirectional. Response: The results show that the risk of both depression and GERD are associated with prior sleep disorders.

Comment: Paragraph 2, page 12: Please rewrite the sentence starting with "Key strengths..." into "Major strengths of this study include the large sample sizes used based on the THIN database...". 
Response: We have now made this change

Comment: Rewrite “...case ascertainment bias was minimized...".

Response: We have now made this change

Conclusions:

No comment.

Reviewer: 3

Comment: This is a well written, clearly presented paper reporting on the relationship of recorded diagnoses of and prescriptions for depression to the subsequent diagnosis/onset of gastro-oesophageal reflux disease (GERD), using the General Practitioner Research Database as the data source. The introduction, methods and results sections are well presented and clear, and I did not find any serious problems with them. However, there are I think one or two further analyses that might have been considered to try to begin to tease out some of the possible mechanisms involved. The first is to look at consultation rates in the depressed and non-depressed (control) subjects. It is conceivable that, because of more frequent contact with services as a result of being depressed, other problems, perhaps rather ill-defined previously, become crystallised and take on diagnostic significance. Vague indigestion/reflux symptoms could be one of these, leading to a diagnosis of GERD appearing in the medical record. This needs to be considered and discussed. Response: We used The Health Improvement Network rather than the GPRD. We agree that more frequent use of healthcare services may result in increased diagnostic rates. However, the risk estimates in the present study were adjusted by consultation rate so the observed associations are unlikely to be solely dependent on consultation behaviour. This is examined in the Discussion (page 11). 
Comment: Second, because one of the criteria for causality is a dose response relationship, it might be worth looking, if possible, in a little more detail at both the duration/chronicity of depression and the intensity/volume of anti-depressant prescribing. The finding that SSRI prescription does not seem to be related to GERD in the same way that tricyclic anti-depressant prescribing is might, perhaps, be explored a little further - were the TCA patients (taking a rather old-fashioned anti-depressant) chronic depressives, and were the SSRI patients more recentlydiagnosed/shorter-term depressives.

Response: Information on the severity of depression is not recorded in a systematic way in the database. We studied first recorded diagnoses of depression, which included a range of disorders: from mild or time-limited conditions, such as postnatal depression or depressed mood, to severe and long-lasting episodes of depression. For our previous study we created a "proxy" measure of depression severity based on treatment duration, recorded diagnostic code and healthcare use. Patients were categorized as less severe when they had no definitive diagnosis recorded (just recorded symptoms of depression), had no specific treatment (antidepressant, anxiolytic or hypnotic drugs) during the 3 months after diagnosis depression or were treated for less than 15 days, and there was only one new PCP visit during the 3 months after diagnosis. The remaining cases were considered to be more severe. The categorization of patients with depression according to this classification showed that patients with more severe depression were not at higher risk of a GERD diagnosis than patients with less severe depression. This is now stated in the results and discussion sections of the current manuscript.

We have also now assessed the association between the time since depression diagnosis and the risk of a GERD diagnosis. Patients who had been diagnosed with depression less than 1 year before the index date had a slightly higher risk of GERD (OR: 1.55; 95\% CI: 1.36-1.76) than those who had been diagnosed with depression more than 1 year before 
the index date (OR: $1.21 ; 95 \%$ CI: 1.10-1.34). To explore if duration of depression (more/less recently diagnosed) could confound the association between the use of antidepressant drugs and development of GERD we further adjusted the multivariable model to include the duration of disease. No major differences were found.

We have now added a sentence in the Results regarding duration and a paragraph at the end of Discussion that examines the other suggested issues.

Comment: In the discussion I think that the paper would benefit from more informed speculation about possible hypotheses beyond the effect of some antidepressants (notably venlaflaxine) on the lower oesophageal sphincter. Are there other possible mechanisms at play and what is the message for clinicians, if any?

Response: We agree with the reviewer that other possible hypotheses may explain the development of GERD among patients exposed to antidepressants. However, we feel that the data from our observational study do not able us to speculate further on pharmacological and clinical issues that are not well established. Nevertheless, as you can see in the following table we observed a non-significant increase in the risk of a GERD diagnosis associated with use of venlafaxine.

\begin{tabular}{|c|c|c|c|c|c|c|}
\hline & \multicolumn{6}{|c|}{ Overall } \\
\hline & \multicolumn{2}{|c|}{ Controls } & \multicolumn{2}{|c|}{$\begin{array}{c}\text { GERD } \\
\text { Cases }\end{array}$} & $\mathbf{O R}^{\mathrm{a}}$ & $95 \% \mathrm{CI}$ \\
\hline & 10000 & $\%$ & 3064 & $\%$ & 2 & \\
\hline Non user & 9588 & 95.9 & 2875 & 93.8 & 1 & - \\
\hline Current & 108 & 1.1 & 54 & 1.8 & 1.33 & $0.95-1.86$ \\
\hline Recent & 114 & 1.1 & 47 & 1.5 & 1.16 & $0.82-1.64$ \\
\hline \multirow[t]{3}{*}{ Past } & 190 & 1.9 & 88 & 2.9 & 1.28 & $0.98-1.66$ \\
\hline & \multicolumn{6}{|c|}{ Only in patients with depression } \\
\hline & 4785 & $\%$ & 1854 & $\%$ & & \\
\hline Non user & 4386 & 91.7 & 1676 & 90.4 & 1 & - \\
\hline Current & 106 & 2.2 & 54 & 2.9 & 1.24 & $0.88-1.73$ \\
\hline Recent & 113 & 2.4 & 46 & 2.5 & 1.04 & $0.73-1.48$ \\
\hline Past & 180 & 3.8 & 78 & 4.2 & 1.07 & $0.81-1.41$ \\
\hline
\end{tabular}




\begin{abstract}
${ }^{a}$ Adjusted by sex, age, body mass index, calendar year, smoking, alcohol use and number of visits to a primary care physician in the previous year.
\end{abstract}

2

4

5

7

8
9

10

11

12

13

14

15

16

17

18

19

20

21

22

23

24

25

26

27

28

29

30

31

32

33

34

35

36

37

38

39

40

41

42

43

44

45

46

47

48

49

50

51

52

53

54

55

56

57

58

59

60 


\section{Depression and treatment with antidepressants are associated with the development of gastroesophageal reflux disease}

Elisa Martín-Merino,* Ana Ruigómez,* Luis A. García Rodríguez,* Mari-Ann Wallander, ${ }^{\dagger}$ Saga Johansson ${ }^{\ddagger} \S$

*Spanish Centre for Pharmacoepidemiologic Research (CEIFE), Madrid, Spain;

†Department of Public Health and Caring Sciences, Uppsala University, Uppsala, Sweden; ${ }^{\ddagger}$ AstraZeneca R\&D Mölndal, Mölndal, Sweden; ${ }^{\S}$ Institute of Medicine, Sahlgrenska Academy, Gothenburg University, Gothenburg, Sweden.

Correspondence to: Ms. E. Martín-Merino, Centro Español de Investigación

Farmacoepidemiológica (CEIFE), C/Almirante 28, 2 28004 Madrid, Spain.

E-mail: elisaceife@ telefonica.net

Short running title: Depression and gastroesophageal reflux disease

Keywords: antidepressants, depression, gastroesophageal reflux disease, GERD, reflux

Word count: 2750 


\title{
Summary
}

Background:The roles of depression and antidepressants in triggering reflux symptoms are unclear.

\begin{abstract}
Aim:To compare the incidence of gastroesophageal reflux disease (GERD) in individuals with and without a depression diagnosis, and to evaluate risk factors for a GERD diagnosis. The relationship between antidepressant treatment and GERD was also assessed.
\end{abstract}

Methods:The Health Improvement Network UK primary care database was used to identify patients with a incident depression diagnosis and an age- and sex-matched control cohort with no depression diagnosis. Incident GERD diagnoses were identified during a mean follow-up of 3.3 years. Furthermore, we performed nested case-control analyses where odds ratios (OR) with $95 \%$ confidence intervals (CI) were estimated by unconditional logistic regression in multivariable models.

Results:The incidence of GERD was 14.2 per 1000 person-years in the depression cohort and 8.3 per 1000 person-years in the control cohort. The hazard ratio of GERD in patients with-a depression diagnosis compared with controls was 1.72 (95\% CI:1.60-1.85). Among patients with depression, tricyclic antidepressant (TCA) use was associated with an increased risk of a GERD diagnosis (OR:1.71; 95\% CI:1.34-2.20), while selective serotonin reuptake inhibitors were not associated with GERD.

Conclusions:A depression diagnosis is associated with an increased risk of a subsequent GERD diagnosis, particularly in individuals using TCAs. 


\section{Introduction}

Gastroesophageal reflux disease (GERD) is a chronic disease ${ }^{1}$ that accounts for approximately $5 \%$ of all primary care consultations. ${ }^{2}$ The prevalence of GERD varies in different parts of the world; ${ }^{3}$ it affects $10-20 \%$ of adults in Europe and the USA, but only about $5 \%$ of adults in Asia. ${ }^{4}$

Depression is also widely reported in primary care, and the prevalence of depression is approximately $11 \%$ in UK primary care. ${ }^{5}$ Several studies have found an association between GERD and depression. ${ }^{6-8}$ In addition, some medications used in psychiatric or psychological disorders (such as hypnotics, antipsychotics and antidepressants) may affect lower esophageal sphincter (LES) tone, salivation or esophageal motility, and thus exacerbate reflux symptoms. ${ }^{9}$

In a previous study, we showed that individuals with a diagnosis of GERD are at risk of a subsequent diagnosis of depression. ${ }^{5}$ The current study aims to evaluate the potential roles of depression and treatment with antidepressants in triggering reflux symptoms. The Health Improvement Network (THIN) UK primary care database was used to compare the incidence of GERD in a cohort of patients with a diagnosis of depression with that in a cohort of individuals with no such diagnosis. Other potential risk factors for a diagnosis of GERD, including the use of antidepressants and comorbidity status, were also evaluated. 


\section{Materials and Methods}

\section{Source population and case ascertainment}

THIN has previously been validated for epidemiological research. ${ }^{10}$ This database contains patient information such as demographic factors, consultation rates, referrals, hospitalizations, laboratory test results and prescriptions ordered by primary care practitioners (PCPs). Diagnoses and test procedures are recorded using Read codes. When prescriptions written by PCPs are generated, they are recorded automatically in the database using a coded drug dictionary (Multilex).

In a previous study, ${ }^{5}$ we identified a cohort of patients (aged 10-79 years) with an incident diagnosis of depression from 2002 to 2004 and an age- and sex-matched control cohort with no diagnosis of depression. Detailed descriptions of cohort ascertainment and diagnosis validation were described in that study. ${ }^{5}$

In the present study, we further excluded from the original cohorts those patients with a recorded diagnosis of GERD or heartburn prior to the study start date (date of depression diagnosis in the case cohort or a randomly generated date in the control cohort; Figure 1). The two cohorts were followed up from the start date until the earliest occurrence of one of the following endpoints: first recorded GERD diagnosis; reaching the age of 80 years; death; or the end of the study period (31 December 2006). All individuals in the control cohort who were diagnosed with depression during the follow-up period were censored at that date, to ensure that the control cohort remained free of depression diagnoses. 
After manual review of the records of all patients with diagnostic codes suggestive of GERD during the follow-up period, 3064 individuals were confirmed as having a diagnosis of GERD (1854 in the depression cohort and 1210 in the control cohort). Previous studies ${ }^{5,11}$ reported that GERD and depression diagnoses have confirmation rates of $73 \%$ and $90 \%$, respectively, after validation by PCPs.

A nested case-control study was also performed to adjust the risk of GERD associated with depression by potential confounding variables such as medication use (Figure 1). We used as cases all identified patients in both cohorts with an incident diagnosis of GERD ( $\mathrm{n}=3064)$. The controls were 10000 age-, sex- and calendar-year-matched individuals with no GERD diagnosis that were sampled from both cohorts. The index date was the date of GERD diagnosis in the cases and a random date during the study period in the controls.

To ascertain the association between antidepressant treatment among patients with depression, a second nested case-control study was carried out using only the cases $(\mathrm{n}=1854)$ and controls $(\mathrm{n}=4785)$ in the depression cohort.

\author{
Statistical Analysis \\ Time-to-event analysis was performed to assess the cumulative incidence of GERD in \\ each cohort during the follow-up period. Cox regression analysis was used to estimate the \\ hazard ratio (HR) of GERD in patients with a diagnosis of depression compared with \\ individuals with no depression diagnosis adjusted by age and sex.
}


Furthermore, we performed unconditional logistic regression in both case-control analyses to estimate odds ratios (ORs) and 95\% confidence intervals of GERD associated with potential risk factors. STATA64 version 10.1 (StataCorp) was used for all the statistical analysis.

For both case-control studies we analysed the following potential risk factors: prior morbidity status, medication use, and demographic and lifestyle factors recorded in the database. Data on sex, age, smoking status, body mass index (BMI), alcohol consumption, prior morbidities and medication status were collected from THIN at the index date. Use of healthcare services between the start date and the index date was also assessed. Prior morbidities were identified by examining the database for Read codes suggestive of cardiovascular, gastrointestinal, inflammatory and respiratory diseases, psychiatric and psychological disorders, hyperlipidaemia, diabetes, pain and cancer. Multilex codes recorded in the database were used to collect data on various drug treatments, including analgesics, anti-inflammatory drugs, drugs affecting the central nervous system, drugs used to treat cardiovascular diseases or metabolic disorders, hormones and acid-suppressing drugs. Therapeutic drug exposure was divided into four categories: 'current users', whose supply of medication from the most recent prescription lasted until the index date or ended in the 30 days before the index date; 'recent users', whose most recent supply lasted until 31-365 days before the index date; 'past users', whose medication ended more than 1 year before the index date; and 'non-users', who had no prescription recorded before the index date. 


\section{Results}

We identified 1854 patients with a new diagnosis of GERD in the depression cohort and 1210 in the control cohort during a mean follow-up of 3.3 years (range: $0-5$ years).

The overall incidence of GERD was 14.2 per 1000 person-years in the depression cohort (95\% confidence interval [CI]: 13.6-14.8) and 8.3 per 1000 person-years in the control cohort (95\% CI: 7.9-8.8). The incidence of GERD increased with age in both cohorts (Figure 2). Although the overall incidence was almost equivalent in men and women, women younger than 30 years had a higher incidence of GERD than men of the same age. This difference was particularly striking in the depression cohort (women: 13.9 per 1000 person-years; men: 7.8 per 1000 person-years).

The age- and sex-adjusted HR of GERD among patients with a diagnosis of depression compared with individuals with no depression diagnosis was 1.72 (95\% CI: 1.60-1.85). The increased risk of GERD in the depression cohort was present throughout the 5 years of follow-up. However, patients who had been diagnosed with depression less than 1 year before the index date had a slightly higher risk of GERD (OR: 1.55; 95\% CI: 1.36-1.76) than those who had been diagnosed with depression more than 1 year before the index date (OR: 1.21; 95\% CI: 1.10-1.34). No relationship was found between the severity of depression and the risk of a GERD diagnosis (data not shown). 
Among patients with depression, the incidence of GERD did not vary by treatment status when all types of medical treatment were taken as a whole. The incidence of GERD among patients with depression who were medically treated within 30 days of diagnosis was 14.4 per 1000 person-years (95\% CI: 13.7-15.2), while the incidence among nontreated patients with depression was 13.2 per 1000 person-years (95\% CI: 11.9-14.6), resulting in a hazard ratio between these two groups of 1.06 (95\% CI: 0.95-1.20). This lack of difference between treated and non-treated patients with depression was further analysed by examining the interaction between depression and antidepressant use in an multivariable unconditional logistic regression model. Using as the reference group individuals with no depression diagnosis who had never been exposed to antidepressants, the OR of GERD associated with a diagnosis of depression (with no medical treatment) was 1.50 (95\% CI: $1.26-1.78)$ and the OR for patients using antidepressant drugs but who had no recorded depression diagnosis was 1.52 (95\% CI: 1.12-2.06).

After adjusting by sex, age, BMI, calendar year, smoking, alcohol use and PCP visits, individuals with a diagnosis of depression (treated or untreated) had a $31 \%$ increase in the risk of a GERD diagnosis compared with individuals with no depression diagnosis (OR: 1.31; 95\% CI: 1.20-1.43) (Table 1). Further adjustment by the presence of painful conditions and the use of antihypertensive drugs, nitrates and non-steroidal antiinflammatory drugs (NSAIDs) reduced this association, although it remained significant (OR: 1.24; 95\% CI: 1.14-1.36). Other risk factors for a diagnosis of GERD are detailed in Table 1. In particular, a prior diagnosis of anxiety, sleep disorder or stress was also associated with an increased risk of a GERD diagnosis. These estimates of risk may have 
been greater if the model had not been adjusted by depression status. We also observed an increase in the risk of GERD associated with a history of painful conditions or the use of non-steroidal anti-inflammatory drugs, antihypertensives or nitrates.

\begin{abstract}
Among patients with a diagnosis of depression, those treated with tricyclic antidepressants (TCAs) had an increased risk of a GERD diagnosis compared with nonusers of these drugs (Table 2). This increase in risk remained statistically significant after adjusting for various comorbidities (cardiovascular, gastrointestinal, articular, renal, liver and respiratory diseases, painful conditions, psychological disorders, cancer and diabetes; data not shown) as well as when adjusting for the time elapsed since first diagnosis of depression (data not shown). Too few individuals were prescribed high-dose TCAs to assess whether there was any dose-response relationship. The risk of a GERD diagnosis did, however, increase with increasing duration of TCA use (OR: 1.48; 95\% CI: $1.07-$ 2.06 for durations of $\leq 3$ months vs OR: 2.06 ; $95 \%$ CI: $1.43-2.97$ for $>3$ months).
\end{abstract}

The most commonly prescribed TCA was amitriptyline, which was prescribed to 58 individuals $(3 \%)$ in the GERD cohort and $92(2 \%)$ in the control cohort. Use of amitriptyline was associated with a $71 \%$ increase in the risk of a GERD diagnosis compared with non-use (OR: 1.71; 95\% CI: 1.22-2.40). This increase in risk was found among long-term (> 3 months) amitriptyline users (OR: 2.19; 95\% CI: 1.32-3.64), but not among short-term ( $\leq 3$ months) users (OR: 1.40; 95\% CI: 0.89-2.22). The use of other TCAs was associated with a non-significant increase in the risk of a GERD diagnosis (dothiepin: OR: 1.40; 95\% CI: 0.89-2.19; lofepramine: OR: 1.39; 95\% CI: 
0.70-2.78). No association was found between use of selective serotonin reuptake inhibitors (SSRIs), serotonin-noradrenalin reuptake inhibitors (SNRIs) or hypnotics and the diagnosis of GERD.

Patients treated with antipsychotics (OR: 1.45; 95\% CI: 1.09-1.92) or anxiolytics (OR: 1.66; 95\% CI: 1.19-2.32) were at an increased risk of a GERD diagnosis, compared with non-users. These small increases in risk were still present after adjusting for various comorbidities (antipsychotics, OR: 1.37; 95\% CI: 1.03-1.82 and anxiolytics, OR: 1.48; 95\% CI: 1.05-2.09).

\section{Discussion}

This study showed that, in UK primary care, patients with a diagnosis of depression are more likely to receive a subsequent diagnosis of GERD than individuals without a depression diagnosis. An association between depression and GERD has been reported previously, ${ }^{6-8}$ but, to our knowledge, ours is the first study to add a temporal aspect to this association.

We observed that depression and treatment with antidepressants are both independently associated with the development of GERD. The risk of a GERD diagnosis was highest in the first year after the depression diagnosis. We found that the risk of GERD in patients with depression was similar in patients who were treated with antidepressant drugs and in those who were not treated with such drugs. Furthermore, we observed that patients who were using antidepressant drugs but who had no recorded depression diagnosis (who 
were presumably using the drugs for another indication) also had an increased risk of a GERD diagnosis.

Patients diagnosed with depression who were taking TCAs, antipsychotics or anxiolytics were at higher risk of a GERD diagnosis than patients with depression who were not taking these medications. It has been shown that drugs with an anticholinergic effect, such as TCAs and antipsychotics, can lead to a decrease in LES pressure and thus an increase in the number of reflux episodes. ${ }^{9}$ Furthermore, anticholinergic medications have been reported to delay gastric emptying, and to inhibit esophageal peristalsis and salivary secretion. ${ }^{12,13}$ A previous study found an association between TCA use and reflux esophagitis that, when stratified by the individual TCA used, was significant only for clomipramine. ${ }^{12}$ Unfortunately, we were unable to test for an association between clomipramine and GERD in the present study due to the small number of patients who were treated with that drug. We also found that benzodiazepines used as anxiolytics were associated with an increased risk of GERD. Previous studies have also shown that benzodiazepines can induce gastroesophageal reflux, probably through reducing LES pressure by acting as a smooth muscle relaxant. ${ }^{14-16}$

In contrast to the association observed between TCA use and GERD, we found no significant association between GERD and the use of SSRIs (the most widely used antidepressant drug class). It is possible that this is due to differences in the anticholinergic effects of these drug classes: SSRIs have negligible to minimal anticholinergic effects while TCAs have a mild to moderately-severe one. ${ }^{17}$ Whether the 
difference in mechanisms between SSRIs and TCAs could explain the different effect on GERD deserves further investigation. The difference in association could also be due to differences in the types of patients who are prescribed TCAs or SSRIs. ${ }^{18}$ However, the multivariable model used in this study should have minimized any such confounding.

A correlation between depression severity and reflux symptom intensity has been proposed by several studies. ${ }^{6,19}$ In interpreting our results, it should be noted that we examined only the first recorded diagnosis of depression for each patient. This diagnosis could cover a range of disorders: from mild or time-limited conditions, such as postnatal depression or depressed mood, to severe and long-lasting episodes of depression. However, we created an algorithm of depression severity using the recorded depression code, the duration of treatment and the number of PCP visits for depression. The categorization of patients with depression according to this classification showed that patients with more severe depression were not at a higher risk of GERD than those with less severe depression.

We found that individuals with a diagnosis of another psychiatric condition such as anxiety or stress disorder were at increased risk of a subsequent diagnosis of GERD (regardless of their depression status) compared with individuals without these conditions. This is in agreement with previous studies, suggesting that anxiety is another factor that might increase the risk of GERD. ${ }^{6,20}$ This suggests a possible common pathological link between psychiatric disorders and reflux symptoms that might be exacerbated by particular types of medications. It has been suggested that individuals 
who are anxious and stressed are more likely than those who are not to perceive lowintensity esophageal stimuli as painful reflux symptoms. ${ }^{21,22}$ It should, however, be noted that anxiety is also known to be associated with increased consultation for GERD and dyspepsia. ${ }^{7,23}$ Thus, patients with anxiety and stress disorders, as well as patients with depression, may simply be more likely to consult their PCP about their symptoms and therefore be more likely to receive a GERD diagnosis than patients without psychiatric disorders. However, the risk estimates in the present study were adjusted for consultation rate, so it is unlikely that this association is solely dependent on consultation behaviour.

We have previously shown that a diagnosis of GERD is associated with a significant increase in the risk of a subsequent diagnosis of a sleep disorder. ${ }^{24}$ This is likely to reflect the presence of nocturnal heartburn, which is known to be associated with sleep disorders. ${ }^{25-27}$ The current study shows that the reverse is also true: a diagnosis of a sleep disorder is associated with a significant increase in the risk of a subsequent diagnosis of GERD.

The incidence of GERD in the control group of the present study is slightly higher than that found in a previous database study in UK primary care (4.5 per 1000 person-years), ${ }^{11}$ and also higher than that reported using data from a US health insurance claims database (5.4 per 1000 person-years). ${ }^{28}$ It is possible that this is due to differences in methodology, selection criteria or database characteristics. Although no validation of GERD diagnoses was performed in the present study, which may have led to an overestimation of the incidence of GERD, previous studies have reported high validation rates for GERD and 
depression diagnoses. 5,11

Major strengths of this study include the large sample sizes that it is possible to study using THIN. The representativeness and completeness of the database are also major advantages, as is the reported validity of the GERD and depression diagnoses. However, there are a number of potential limitations to this study. Because of the nature of the database, we were only able to capture individuals who consulted with their PCPs regarding their symptoms and who received a diagnosis of GERD or depression. GERD and depression can be difficult to diagnose, and the diagnostic codes used may vary between PCPs. However, we found no significant difference in the risk of a diagnosis of GERD between patients who were being treated for their depression and those who were not, which suggests that ease ascertainment bias was minimized. misclassification of depression diagnoses was minimized. Another potential limitation is that the indication for the antidepressants studied was not assessed. This may have led to some confounding by indication because TCAs can be used in the treatment of some functional gastrointestinal disorders. ${ }^{29-31}$ However, a previous study in UK primary care found that antidepressants and anxiolytics were only prescribed to $3 \%$ of patients with irritable bowel syndrome ${ }^{32}$ so it is unlikely that any potential confounding would have had a major impact on the results.

\section{Conclusions}

This is the first study to show that a diagnosis of depression is associated with a significant increase in the risk of a subsequent GERD diagnosis. Further research is 
needed to fully elucidate the reasons behind this association. This study provides further evidence for the link between the presence of reflux symptoms and the use of TCAs, anxiolytic and antipsychotic drugs. These factors should be taken into account when managing depression and GERD in primary care. 


\title{
Statement of interests
}

\author{
Authors' declaration of personal interests: \\ Luis A. García Rodríguez, Ana Ruigómez and Elisa Martín-Merino work for CEIFE, \\ which has received research funding from AstraZeneca R\&D Mölndal. \\ Luis A. García Rodríguez has received consultancy fees from AstraZeneca R\&D \\ Mölndal. \\ Saga Johansson is an employee of AstraZeneca R\&D Mölndal, and Mari-Ann Wallander \\ was an employee of AstraZeneca R\&D Mölndal at the time the study was conducted.
}

\section{Declaration of funding interests:}

This study was funded by an unrestricted research grant from AstraZeneca R\&D Mölndal.

Writing support was provided by Catherine Hill of Oxford PharmaGenesis ${ }^{\mathrm{TM}}$ Limited and funded by AstraZeneca R\&D Mölndal. 


\section{References}

1. Armstrong D. Systematic review: persistence and severity in gastroesophageal reflux disease. Aliment Pharmacol Ther 2008; 28: 841-53.

2. Jones R. Gastro-oesophageal reflux disease in general practice. Scand J Gastroenterol Suppl 1995; 211: 35-8.

3. Vakil N, Veldhuyzen van Zanten S, Kahrilas P, Dent J, Jones R. The Montreal definition and classification of gastro-esophageal reflux disease (GERD) - a global evidence-based consensus. Am J Gastroenterol 2006; 101: 1900-20.

4. Dent J, El-Serag HB, Wallander MA, Johansson S. Epidemiology of gastrooesophageal reflux disease: a systematic review. Gut 2005; 54: 710-17.

5. Martín-Merino E, Ruigómez A, Johansson S, Wallander M-A, García-Rodríguez LA. Study of a cohort of patients newly diagnosed with depression in general practice: prevalence, incidence, comorbidity and treatment patterns. Prim Care Companion J Clin Psychiatry 2010; 12: e1-e8.

6. Jansson C, Nordenstedt H, Wallander MA, et al. Severe gastro-oesophageal reflux symptoms in relation to anxiety, depression and coping in a population-based study. Aliment Pharmacol Ther 2007; 26: 683-91.

7. Hu WH, Wong WM, Lam CL, et al. Anxiety but not depression determines health care-seeking behaviour in Chinese patients with dyspepsia and irritable bowel syndrome: a population-based study. Aliment Pharmacol Ther 2002; 16: 2081-8.

8. Avidan B, Sonnenberg A, Giblovich H, Sontag SJ. Reflux symptoms are associated with psychiatric disease. Aliment Pharmacol Ther 2001; 15: 1907-12.

9. Kahrilas PJ. GERD pathogenesis, pathophysiology, and clinical manifestations. Cleve Clin J Med 2003; 70 (Suppl 5): S4-19.

10. Lewis JD, Schinnar R, Bilker WB, Wang X, Strom BL. Validation studies of the health improvement network (THIN) database for pharmacoepidemiology research. Pharmacoepidemiol Drug Saf 2007; 16: 393-401.

11. Ruigómez A, García Rodríguez LA, Wallander MA, Johansson S, Graffner H, Dent J. Natural history of gastro-oesophageal reflux disease diagnosed in general practice. Aliment Pharmacol Ther 2004; 20: 751-60. 
12. van Soest EM, Dieleman JP, Siersema PD, Schoof L, Sturkenboom MC, Kuipers EJ. Tricyclic antidepressants and the risk of reflux esophagitis. Am J Gastroenterol 2007; 102: 1870-7.

13. Spechler SJ. Gastroesophageal reflux disease and its complications. In: Friedman SL, McQuaid KR, Grendell JH, eds. Current Diagnosis and Treatment in Gastroenterology. 2nd ed. New York: McGraw-Hill; 2003. p. 266-82.

14. Hall JA, Magne ML, Twedt DC. Effect of acepromazine, diazepam, fentanyldroperidol, and oxymorphone on gastroesophageal sphincter pressure in healthy dogs. Am $J$ Vet Res 1987; 48: 556-7.

15. Rushnak MJ, Leevy CM. Effect of diazepam on the lower esophageal sphincter. A double-blind controlled study. Am J Gastroenterol 1980; 73: 127-30.

16. Fass R, Quan SF, O'Connor GT, Ervin A, Iber C. Predictors of heartburn during sleep in a large prospective cohort study. Chest 2005; 127: 1658-66.

17. Baldessarini RJ. Drugs and the treatment of psychiatric disorders: depression and anxiety disorders. In: Hardman JG, Limbird L, eds. Goodman and Gilman's the pharmacological basis of therapeutics. 9th ed. New York: McGraw-Hill Professional; 1996.

18. Bauer M, Monz BU, Montejo AL, et al. Prescribing patterns of antidepressants in Europe: Results from the Factors Influencing Depression Endpoints Research (FINDER) study. Eur Psychiatry 2008; 23: 66-73.

19. Lapina NS, Borovkov NN. [Anxious depressive conditions in patients with gastroesophageal reflux disease]. Klin Med (Mosk) 2008; 86: 59-62.

20. Saberi-Firoozi M, Khademolhosseini F, Yousefi M, Mehrabani D, Zare N, Heydari ST. Risk factors of gastroesophageal reflux disease in Shiraz, southern Iran. World J Gastroenterol 2007; 13: 5486-91.

21. Mizyed I, Fass SS, Fass R. Review article: gastro-oesophageal reflux disease and psychological comorbidity. Aliment Pharmacol Ther 2009; 29: 351-8.

22. Bradley LA, Richter JE, Pulliam TJ, et al. The relationship between stress and symptoms of gastroesophageal reflux: the influence of psychological factors. Am J Gastroenterol 1993; 88: 11-9. 
23. Hungin AP, Hill C, Raghunath A. Systematic review: frequency and reasons for consultation for gastroesophageal reflux disease and dyspepsia. Aliment Pharmacol Ther 2009; 30: 331-42.

24. Wallander MA, Johansson S, Ruigómez A, García Rodríguez LA, Jones R.

Morbidity associated with sleep disorders in primary care: a longitudinal cohort study. Prim Care Companion J Clin Psychiatry 2007; 9: 338-45.

25. Shaker R, Castell DO, Schoenfeld PS, Spechler SJ. Nighttime heartburn is an underappreciated clinical problem that impacts sleep and daytime function: the results of a Gallup survey conducted on behalf of the American Gastroenterological Association. Am J Gastroenterol 2003; 98: 1487-93.

26. Orr WC, Heading R, Johnson LF, Kryger M. Review article: sleep and its relationship to gastro-oesophageal reflux. Aliment Pharmacol Ther 2004; 20 (Suppl 9): 39-46.

27. Shaker R, Brunton S, Elfant A, Golopol L, Ruoff G, Stanghellini V. Review article: impact of night-time reflux on lifestyle - unrecognized issues in reflux disease. Aliment Pharmacol Ther 2004; 20 (Suppl 9): 3-13.

28. Kotzan J, Wade W, Yu HH. Assessing NSAID prescription use as a predisposing factor for gastroesophageal reflux disease in a Medicaid population. Pharm Res 2001; 18: 1367-72.

29. Ford AC, Talley NJ, Schoenfeld PS, Quigley EM, Moayyedi P. Efficacy of antidepressants and psychological therapies in irritable bowel syndrome: systematic review and meta-analysis. Gut 2009; 58: 367-78.

30. Jackson JL, O'Malley PG, Tomkins G, Balden E, Santoro J, Kroenke K. Treatment of functional gastrointestinal disorders with antidepressant medications: a meta-analysis. Am J Med 2000; 108: 65-72.

31. Bixquert-Jimenez M, Bixquert-Pla L. [Antidepressant therapy in functional gastrointestinal disorders]. Gastroenterol Hepatol 2005; 28: 485-92.

32. Ruigómez A, Wallander MA, Johansson S, García Rodríguez LA. One-year followup of newly diagnosed irritable bowel syndrome patients. Aliment Pharmacol Ther 1999; 13: 1097-102. 
Table 1. Characteristics of individuals with and without a diagnosis of gastroesophageal reflux disease (GERD), and their association with a diagnosis of GERD

\begin{tabular}{|c|c|c|c|c|}
\hline & $\begin{array}{c}\text { GERD cases } \\
\begin{array}{c}(\mathrm{N}=3064) \\
\mathrm{n}(\%)\end{array}\end{array}$ & $\begin{array}{l}\text { Controls with } \\
\text { no GERD } \\
\text { diagnosis } \\
\begin{array}{c}\text { (N = } 10000) \\
\text { n }(\%)\end{array}\end{array}$ & $\mathbf{O R}^{\mathrm{a}}$ & $95 \% \mathrm{CI}$ \\
\hline \multicolumn{5}{|l|}{ Sex } \\
\hline Female & $1944(63.4)$ & $6356(63.6)$ & NA & - \\
\hline Male & $1120(36.6)$ & $3644(36.4)$ & NA & - \\
\hline \multicolumn{5}{|l|}{ Year of index date } \\
\hline 2002 & $129(4.2)$ & $381(3.8)$ & NA & - \\
\hline 2003 & 418 (13.6) & $1372(13.7)$ & NA & - \\
\hline 2004 & $742(24.2)$ & $2427(24.3)$ & NA & - \\
\hline 2005 & $852(27.8)$ & $2793(27.9)$ & NA & \\
\hline 2006 & $923(30.1)$ & $3027(30.3)$ & NA & \\
\hline \multicolumn{5}{|l|}{ Age (years) } \\
\hline $10-19$ & $103(3.4)$ & $334(3.3)$ & NA & - \\
\hline 20-39 & $1047(34.2)$ & $3426(34.3)$ & NA & - \\
\hline $40-59$ & $1171(38.2)$ & $3829(38.3)$ & NA & - \\
\hline $60-79$ & $743(24.2)$ & $2411(24.1)$ & NA & - \\
\hline \multicolumn{5}{|l|}{ BMI $\left(\mathrm{kg} / \mathrm{m}^{2}\right)^{\mathbf{b}}$} \\
\hline $13-19$ & $173(5.6)$ & $598(6.0)$ & 0.94 & $0.78-1.14$ \\
\hline $20-24$ & $904(29.5)$ & $3129(31.3)$ & 1.00 & - \\
\hline $25-29$ & $866(28.3)$ & $2633(26.3)$ & 1.09 & $0.98-1.22$ \\
\hline$\geq 30$ & $642(21.0)$ & $1615(16.2)$ & 1.23 & $1.09-1.39$ \\
\hline \multicolumn{5}{|c|}{ Alcohol use (units ${ }^{\mathrm{c}}$ per week) } \\
\hline No use & $1174(38.3)$ & $3345(33.5)$ & 1.00 & - \\
\hline $1-15$ & $1130(36.9)$ & $3900(39.0)$ & 0.84 & $0.76-0.93$ \\
\hline $16-42$ & $262(8.6)$ & $756(7.6)$ & 1.00 & $0.85-1.18$ \\
\hline$\geq 43$ & $58(1.9)$ & $106(1.1)$ & 1.39 & $0.99-1.95$ \\
\hline \multicolumn{5}{|l|}{ Smoking status } \\
\hline Never & $838(27.3)$ & $3092(30.9)$ & 1.00 & - \\
\hline Current & $1080(35.2)$ & $3510(35.1)$ & 1.06 & $0.96-1.18$ \\
\hline Former & $1008(32.9)$ & $2713(27.1)$ & 1.22 & $1.10-1.36$ \\
\hline \multicolumn{5}{|l|}{ Healthcare use } \\
\hline Referral or hospitalization & $2217(72.4)$ & $5607(56.1)$ & 1.66 & $1.50-1.84$ \\
\hline
\end{tabular}


762 (24.9)

3339 (33.4)

1.37

1.18-1.59

$\geq 11$

1974 (64.4)

4750 (47.5)

2.61

2.24-3.04

Prior morbidity

Depression

$1854(60.5)$

4785 (47.9)

1.31

$1.20-1.43$

Stress

483 (15.8)

1128 (11.3)

1.19

$1.06-1.35$

Sleep disorder

466 (15.2)

955 (9.6)

1.39

$1.23-1.58$

Anxiety

859 (28.0)

1788 (17.9)

1.46

1.32-1.62

Painful condition

1570 (51.2)

3270 (32.7)

1.80

$1.66-1.97$

Current medication use

NSAIDs

239 (7.8)

605 (6.1)

1.42

1.20-1.69

Antihypertensives

$695(22.7)$

$1781(17.8)$

1.24

$1.09-1.40$

Nitrates

72 (2.3)

133 (1.3)

1.48

1.09-2.00

\footnotetext{
${ }^{a}$ Adjusted by sex, age, BMI, calendar year, smoking, alcohol use, PCP visits and depression status.

${ }^{\mathrm{b}} \mathrm{BMI}$ data missing in 479 GERD cases and 2025 controls.

${ }^{\mathrm{c}}$ One unit of alcohol is equal to $10 \mathrm{~mL}(\sim 8 \mathrm{~g})$ ethanol (alcohol use was not reported by 440 patients in the depression cohort and by 1893 individuals in the control cohort).

${ }^{\mathrm{d}}$ Reference group: 0-2 visits.

NA: Not applicable because of the matched design.

BMI, body mass index; CI, confidence interval; NSAIDs, non-steroidal anti-inflammatory drugs; OR, odds ratio; PCP, primary care practitioner.
} 
Table 2. Current use of medication and its association with a diagnosis of gastroesophageal reflux disease (GERD), compared with non-use, among individuals from the depression cohort with and without a diagnosis of GERD

\begin{tabular}{|c|c|c|c|c|}
\hline & $\begin{array}{c}\text { GERD cases } \\
\begin{array}{c}(\mathrm{N}=1854) \\
n(\%)\end{array}\end{array}$ & $\begin{array}{c}\text { Controls with } \\
\text { depression but } \\
\text { no GERD } \\
\text { diagnosis } \\
(\mathrm{N}=4785) \\
\text { n (\%) }\end{array}$ & $\mathbf{O R}^{\mathbf{a}}$ & $95 \% \mathrm{CI}$ \\
\hline Antidepressants & $550(29.7)$ & $1317(27.5)$ & 1.02 & $0.85-1.24$ \\
\hline $\mathrm{TCA}^{\mathrm{b}}$ & $112(6.0)$ & $182(3.8)$ & 1.71 & $1.34-2.20$ \\
\hline TCA-ser & $94(5.1)$ & $156(3.3)$ & 1.67 & $1.28-2.19$ \\
\hline TCA-nor & $18(1.0)$ & $27(0.6)$ & 1.68 & $0.91-3.09$ \\
\hline SSRI & $381(20.6)$ & $995(20.8)$ & 0.93 & $0.79-1.10$ \\
\hline SNRI & $56(3.0)$ & $113(2.4)$ & 1.20 & $0.86-1.67$ \\
\hline MAOI & $0(0)$ & $1(0.02)$ & - & - \\
\hline Other & $36(1.9)$ & $78(1.6)$ & 1.19 & $0.79-1.78$ \\
\hline Antipsychotics & $81(4.4)$ & $153(3.2)$ & 1.45 & $1.09-1.92$ \\
\hline Anxiolytics & $60(3.2)$ & 93 (1.9) & 1.66 & $1.19-2.32$ \\
\hline Hypnotics & $77(4.2)$ & $200(4.2)$ & 0.97 & $0.74-1.28$ \\
\hline
\end{tabular}

$\overline{{ }^{a} \text { Adjusted by sex, age, body mass index, calendar year, smoking, alcohol use and number }}$ of visits to a primary care physician in the previous year.

${ }^{\mathrm{b}}$ One patient was taking a TCA-ser and a TCA-nor.

CI, confidence interval; MAOI, monoamine oxidase inhibitor; OR, odds ratio; SNRI, selective serotonin and noradrenaline reuptake inhibitor; SSRI, selective serotonin reuptake inhibitor; TCA, tricyclic antidepressant; TCA-nor, tricyclic antidepressant with a predominant action on the reuptake of noradrenaline; TCA-ser, tricyclic antidepressant with an inhibitory action on the reuptake of serotonin. 
Figure 1. Case ascertainment and case-control analysis.

GERD, gastroesophageal reflux disease.

Figure 2. Incidence of gastroesophageal reflux disease stratified by age in a cohort of patients with a diagnosis of depression, and an age- and sex-matched control cohort with no diagnosis of depression. 
Case ascertainment and case-control analysis.

GERD, gastroesophageal reflux disease.

$$
160 \times 91 \mathrm{~mm}(600 \times 600 \mathrm{DPI})
$$




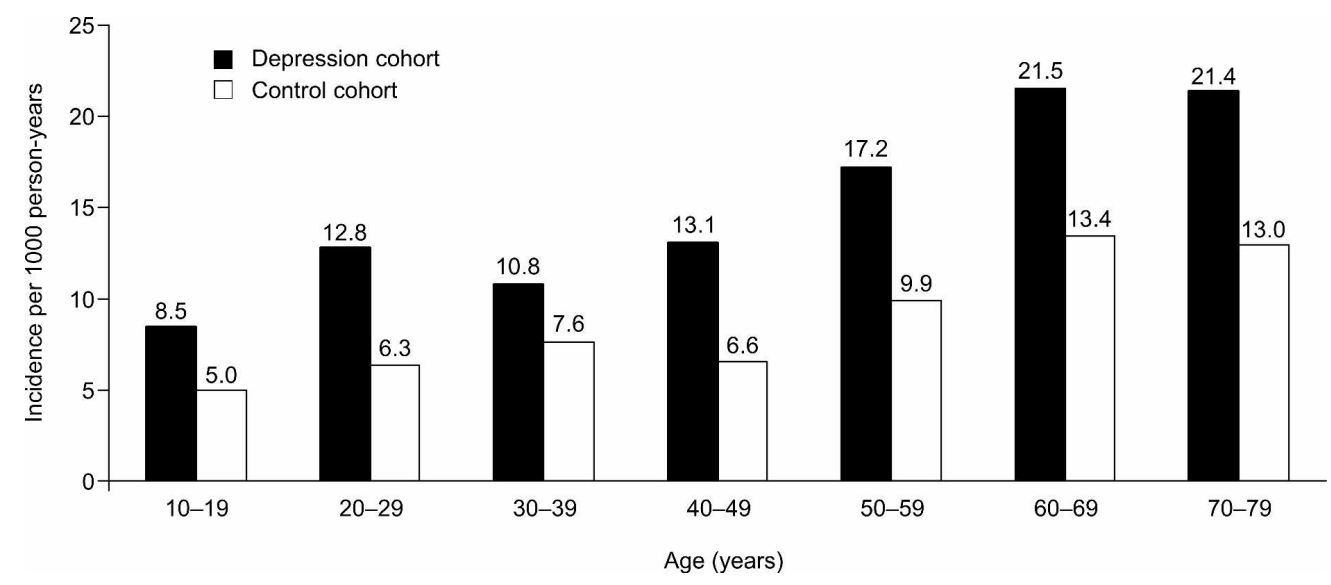

Incidence of gastroesophageal reflux disease stratified by age in a cohort of patients with a diagnosis of depression, and an age- and sex-matched control cohort with no diagnosis of depression.

$158 \times 67 \mathrm{~mm}(600 \times 600 \mathrm{DPI})$ 